\title{
Erratum: A vesicle-trafficking protein commandeers Kv channel voltage sensors for voltage-dependent secretion
}

Christopher Grefen, Rucha Karnik, Emily Larson, Cécile Lefoulon, Yizhou Wang, Sakharam Waghmare, Ben Zhang, Adrian Hills and Michael R. Blatt

Nature Plants 1, 15108 (2015); published online 3 August 2015; corrected 7 October 2015

In the original version of this Article the $y$-axis labels in Figs $2 \mathrm{~b}$,d and $3 \mathrm{f}$ were incorrect. These should have read 'YFP/GFP fluorescence ratio (rel)'. Additionally the Faraday constant $\left(F_{\mathrm{a}}\right)$ was mistakenly omitted from Equation (1). These errors have been corrected. 